\title{
The effect of the resistive properties of bone on neural excitation and electric fields in cochlear implant models
}

\author{
T.K. Malherbe ${ }^{\text {a }}$, T. Hanekom ${ }^{\text {a }}$, J.J. Hanekom ${ }^{\text {a }}$ \\ a Bioengineering, Department of Electrical, Electronic and Computer Engineering, University of \\ Pretoria, Lynnwood Road, Pretoria, Gauteng 0002, South Africa
}

\section{Corresponding Author}

T. Hanekom

Tel.: +27 124202647

Fax: +27123625000

E-mail address: tania.hanekom@up.ac.za

\section{Abstract}

The resistivity of bone is the most variable of all the tissues in the human body, ranging from $312 \Omega . c m$ to $84745 \Omega . c m$. Volume conduction models of cochlear implants have generally used a resistivity value of $641 \Omega . c m$ for the bone surrounding the cochlea. This study investigated the effect that bone resistivity has on modelled neural thresholds and intracochlear potentials using userspecific volume conduction models of implanted cochleae applying monopolar stimulation. The complexity of the description of the head volume enveloping the cochlea was varied between a simple infinite bone volume and a detailed skull containing a brain volume, scalp and accurate return electrode position. It was found that, depending on the structure of the head model and implementation of the return electrode, different bone resistivity values are necessary to match 
model predictions to data from literature. Modelled forward-masked spatial tuning curve (fmSTC) widths and slopes and intracochlear electric field profile length constants were obtained for a range of bone resistivity values for the various head models. The predictions were compared to measurements found in literature. It was concluded that, depending on the head model, a bone resistivity value between $3500 \Omega . c m$ and $10500 \Omega . c m$ allows prediction of neural and electrical responses that match measured data. A general recommendation is made to use a resistivity value of approximately $10000 \Omega . c m$ for bone volumes in conduction models of the implanted cochlea when neural excitation is predicted and a value of approximately $6500 \Omega . \mathrm{cm}$ when predicting electric fields inside the cochlear duct.

\section{Keywords}

Cochlear Implant; Volume Conduction Model; Bone resistivity; FEM; Neural Excitation; Subject Specific; Intracochlear potentials; Skull model; EFI; Forward masked spatial tuning curves

\section{List of abbreviations}

VC Volume Conduction

FE Finite element

GSEF Generalised Schwartz-Eikhof-Frijns

fmSTC Forward masked spatial tuning curve

EFI Electrical field imaging 


\section{Introduction}

Geddes and Baker reported in 1967 that the resistivity of bone is the most variable of all the tissues of the body because of the variation in its composition throughout the body (Geddes and Baker,

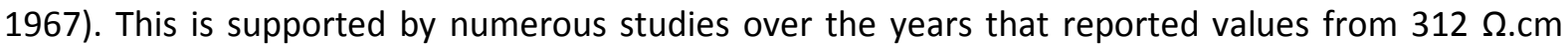

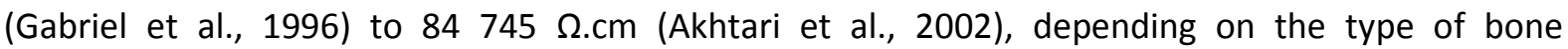
(cancellous vs. cortical), orientation of the bone sample during measurement, measurement frequency, species, state of the bone (e.g. live vs. dried and rehydrated) and the specific site, e.g. skull vs. tibial bone.

Volume conduction (VC) modelling studies of the distribution of currents as a result of intracochlear stimulation with cochlear implant electrodes have conventionally used a homogeneous, isotropic, purely resistive value to represent the electrical characteristics of bone. However, since the compact bone that envelops the human cochlea is mainly responsible for directing current through the cochlear structures instead of allowing dispersion of the currents throughout the surrounding head tissues, it is hypothesised that its electrical properties will have a significant effect on the excitation profiles of the auditory neurons. This effect is especially significant using monopolar stimulation where the return electrode is located outside the cochlea in the surrounding bone. The human cochlea is enveloped in what is regarded as some of the densest bone in the human body. Although bone density is not a good indicator of the absolute resistivity of cortical bone, it is reported to be less conducting than cancellous bone. Bone density is affected by many factors including age, chemical composition, gender and disease, e.g. otosclerosis (Bozorg Grayeli et al., 2004), and varies among individuals (Marshall et al., 1996).

Some VC models of the cochlea have thus far used a value of $641 \Omega . \mathrm{cm}$ for the resistivity of the bone surrounding the vestibular duct (Frijns et al., 1995; Finley et al., 1990; Hanekom, 2001b; Malherbe et

\footnotetext{
${ }^{1}$ Abbreviations: Volume Conduction (VC), finite element (FE), generalised Schwartz-Eikhof-Frijns (GSEF), forward masked spatial tuning curve (fmSTC), electrical field imaging (EFI)
} 
al., 2013). This value originated from a value reported in 1987 by Spelman and Clopton (cited in Finley et al. (1990)) and was derived from guinea pig experiments. However, absolute thresholds predicted for humans using this value are greatly overestimated (Hanekom, 2001a; Briaire and Frijns, 2006), while some animal models predict relatively accurate thresholds using this value, e.g. the guinea pig model of Govindasamy (2012). Other studies on neural excitation in and around the

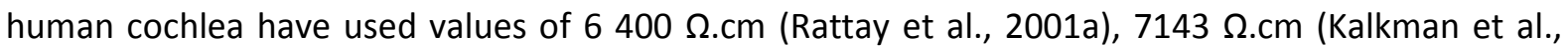

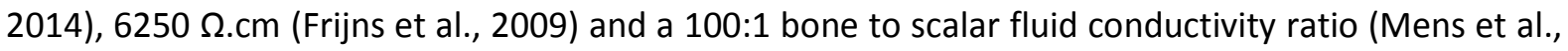
1999; Whiten, 2007) which equates to around $7042 \Omega . \mathrm{cm}$ in the present study. These values were mainly derived by comparing modelled results to objective data. These values are an order larger than the value used in the other studies mentioned. Such large variability of values complicates the selection of the resistivity value to use in a VC model.

The objective of this communication is to report the effect of bone resistivity variations on neural excitation spread and intracochlear potential spread predictions that use VC models as their premise and to propose a range of values that provide realistic predictions when all other tissue resistivities that are used in present VC models of the cochlea are assumed to be sufficiently accurate. The report also deals with various levels of complexity of the implementation of the head volume surrounding the cochlea to assist modellers in making an appropriate choice for the bone resistivity value based on the structure of their model. Forward-masked spatial tuning curve (fmSTC) widths and slopes and electric field profile length constants are compared to measured data found in literature to assess the validity of a predicted result for a bone resistivity value.

\section{Methods}

Volume conduction models of the implanted cochlea in different configurations were used to assess the effect that the value of bone resistivity has on the spread of neural excitation and intracochlear 
electric fields. In all model configurations the bone resistivity value was varied while neural and electric spread was calculated. Spread was measured in the form of width $(\mathrm{mm})$ and slope $(\mathrm{dB} / \mathrm{mm})$ of the neural excitation curves and in the form of length constant of the intracochlear electric field profiles. These neural and electric spread predictions were then compared to data from literature to determine appropriate bone resistivity values to be used in cochlear models.

The model predicted neural excitation spread from the present study was compared to data in a study from Nelson et al. (2008) in the form of fmSTCs. The average monopolar spread for six implanted Clarion HiFocus users was estimated. The fmSTC slope of $1.2 \mathrm{~mm} / \mathrm{dB}$ which they obtained was used as the benchmark for the predictions in the present study. Their study also measured the average fmSTC width at $1 \mathrm{~dB}$ above threshold as $4.6 \mathrm{~mm}$, which was used as the benchmark to which the results in the present study were compared to. Care was taken to mimic the methodology of the Nelson et al. experiments to ensure that the data is comparable.

The electric field data from literature came from a study by Tang et al. (2011) where electrical field imaging (EFI) data of five implanted cochleae are presented. An EFI curve represents the voltages measured on all the electrodes of an implanted array when a stimulus is presented through a single electrode. In that study EFI profiles were obtained for a basal, middle and apical stimulus electrode in each of five implanted ears. The averaged EFI profiles of these electrodes in all the ears were compared to the modelled data in the present study. All the ears in the Tang et al. (2011) study were implanted with Clarion HiFocus electrode arrays; subsequently the volume conduction models in the present study were also implemented with Clarion HiFocus electrode arrays.

\subsection{Volume conduction models}

Five finite element (FE) volume conduction (VC) models based on the morphologies of five individual implanted cochleae of live human implantees were used. All ears of the users have been implanted 
with the Nucleus 24 cochlear prosthesis from Cochlear Limited: four with contour electrode arrays and one with a straight electrode array. However, although the Nucleus device allows the recording of electrode potentials, a floating reference ground causes difficulties in obtaining absolute potential levels and thus electric field profiles cannot be measured for Nucleus users. To compare modelled results to the Nelson et al. data in the neural domain (fmSTCs) and to the Tang et al. (2011) data in the electrical domain (electric field profiles), it was thus necessary to convert the Nucleus electrode arrays in the VC models to Clarion HiFocus electrode arrays. This was done by changing the size and spacing of the modelled electrode contacts to those of a HiFocus array while maintaining the intra scalar location of the electrode carrier of each user. This conversion resulted in the electrode contacts having a slightly curved surface as opposed to a flat surface of a HiFocus array. The effect of this slight curvature on results was assumed to be minimal as the surface area of the HiFocus electrodes was maintained.

The geometry of each model was constructed from computed tomography (CT) data of each implanted ear using similar methodology as described for the construction of our guinea pig model (Malherbe et al., 2013). In that study, the bony geometry of the cochlea and location of the electrode contacts were estimated from $\mu$-CT data and augmented with a model containing the finer inner structures of the cochlea. The same approach was followed in the present models, with the exception being that the bony cochlear geometry was estimated from relatively low resolution standard clinical CT images of which the image sharpness was increased using bicubic interpolation and application of a colour lookup table. Figure 1 shows a mid-modiolar section through a single duct of a user's cochlear model with regions that have different material properties (electrical resistivity) indicated. The material that envelops the structures is bone and is indicated in grey. The spiral lamina is also represented by the same material property as that of the enveloping bone volume (see Figure 1). This assumption is based on the observation that the spiral lamina is an extension of the bone that envelops the cochlear canals. The material properties are the same as those used in the guinea pig model with the exception of the bone value that is being varied. 


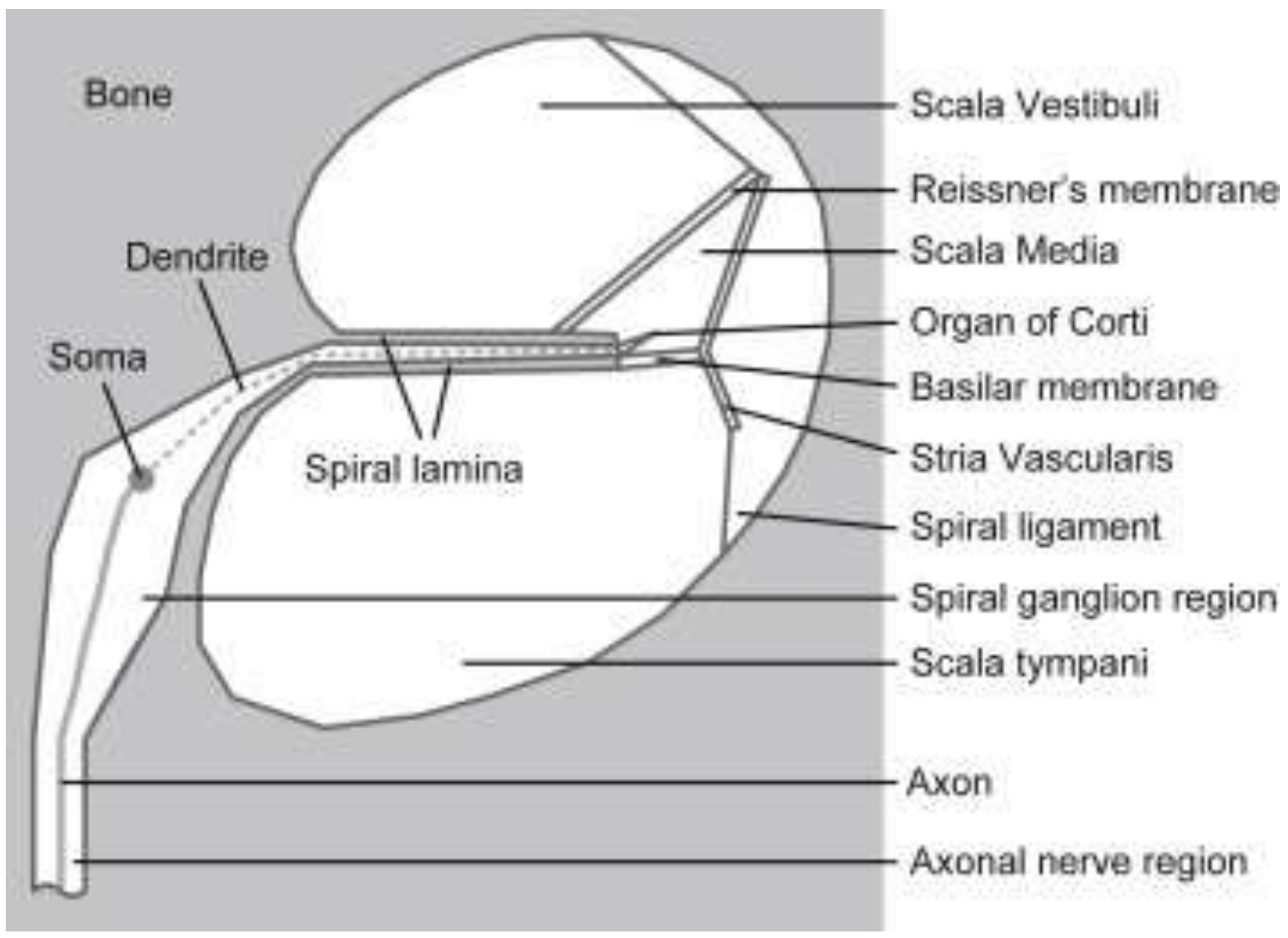

Fig. 1.

Mid-modiolar section through a single duct of the cochlear part of the VC model indicating modelled structures that were assigned different material properties. Modelled structures are entirely encased in bone (grey area). The spiral lamina is also modelled with the same material as the surrounding bone. The position of the neuron is indicated with the soma in the spiral ganglion region. 
Three levels of complexity of the description of the head volume enveloping the cochlea were implemented in conjunction with the user-specific cochlear models described in the previous paragraph. This is important since the structure and materials comprising the head volume affect the current paths, especially in the case of monopolar stimulation. The first type of head model, called the infinite bone model (Figure 2a), contained a rudimentary description of the head consisting of an infinitely large homogeneous bone volume with the user-specific cochlear model at the origin. A sphere with finite dimensions was modelled with its boundaries moved to a virtual distance of infinity using the FEM software package Comsol Multiphysics 4.4. For monopolar stimulation, the return electrode was defined as the entire outer surface of the infinite sphere. This configuration represents the simplest implementation of the head volume and is typically used by modellers for its ease of implementation.

The second type of head model, called the elliptical return electrode model (Figure $2 b$ ), aimed to implement an accurate return electrode. This was done by embedding the cochlear structures into an elliptical homogeneous purely resistive bone volume approximately the shape and size of a full human head (measured crown to chin, ear to ear and nose to back of head from CT data). The position of the return electrode (casing) was estimated from the CT data and placed in the model in the position in which it occurs relative to the cochlea in each user close to the exterior boundary of the bone volume. The accurate return electrode implementation allows for a more realistic representation of the current paths from the cochlea than the infinite bone model whilst being relatively simple to implement. 
The third type of head model, which is the most complex implementation of the tissues enveloping the cochlea, was represented by a detailed skull containing a brain volume and scalp. The skull was constructed from CT scan data of a human head with the return electrode placed in a location specific to each user. This type of model, called the skull model (Figure 2c), did not reflect any cancellous bone volumes that might be present in the temporal bone in the vicinity of the cochlea. The brain volume was assigned a resistivity equal to the average of grey and white matter (500 S.cm) (Haueisen et al., 1997) and the scalp a resistivity of 0.33S/m (Rush and Driscoll, 1968). The outer surfaces of the skull model and the elliptical return electrode model were set up as insulators to resemble the models being in a highly non-conductive medium such as air.

In total 15 models were generated, i.e. the five user-specific cochlear models were embedded into the infinite bone, elliptical return electrode and skull models. In each model the resistivity of the bone volumes was adjusted from $641 \Omega . \mathrm{cm}$ (the value that is traditionally used in VC models) to $26546 \Omega . c m$ (which approximates the value reported by Akhtari in 2002 for compact live skull bone) at intervals as reported in Table 1. A value of $2000 \Omega$.cm was included to allow for continuity across the resistivity range. The VC model was solved for a direct current injected through an electrode to produce a potential distribution throughout the 3D model. This was done for each electrode with each bone resistivity value for each user-specific cochlear model in each of the three head models. Electrode and neural node voltages were then extracted from these potentials and processed further to obtain fmSTCs and electric field profiles. 
a) Infinite bone model

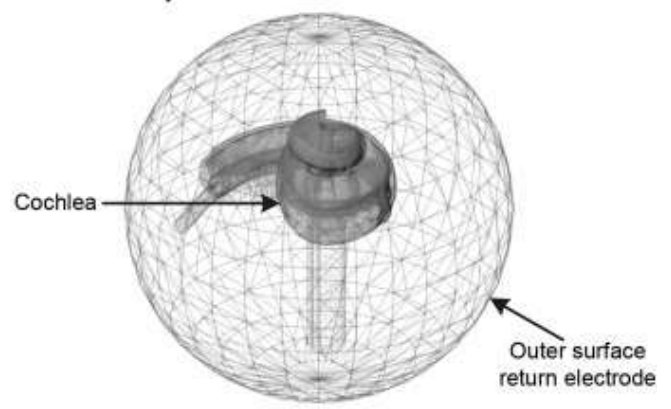

b) Elliptical return electrode model

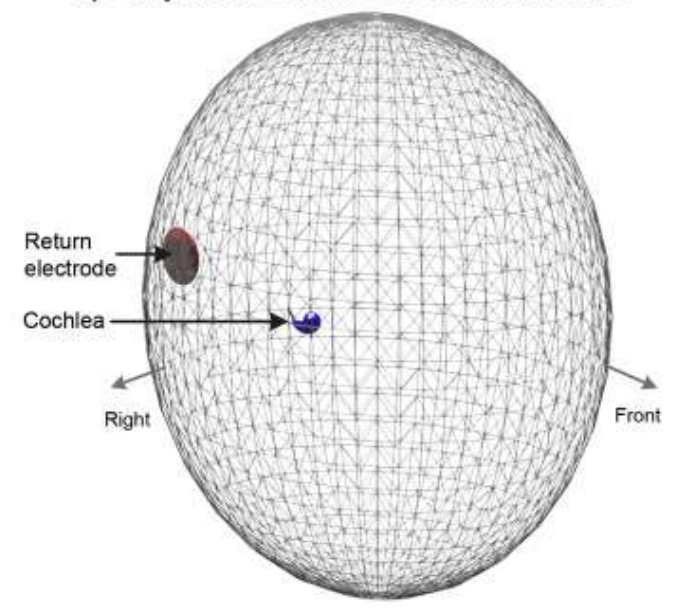

c) Skull model

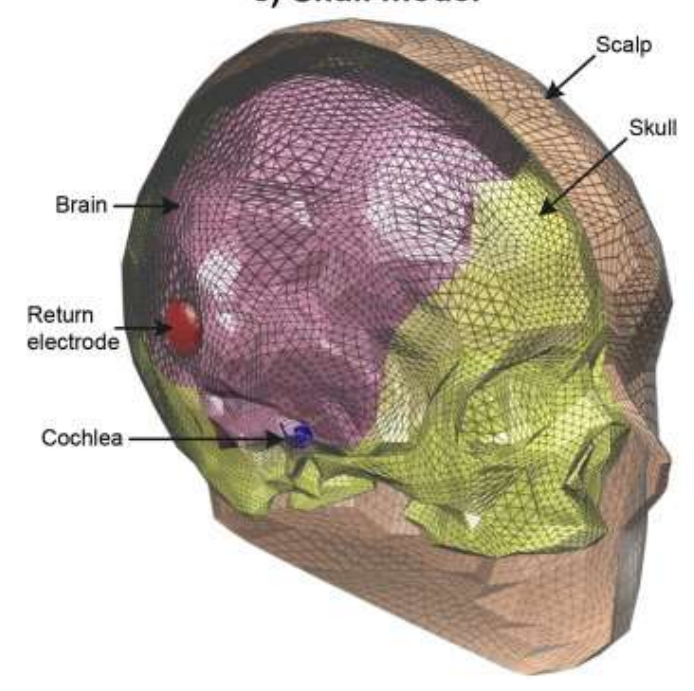

Fig. 2.

Three levels of complexity of the description of the head volume enveloping the cochlea were implemented to investigate the effect of model structure on the bone resistivity. a) Infinite bone model: The cochlea is modelled in an infinitely large bone sphere (see text) with the entire outer surface as the return electrode. b) Ellipticalreturn electrode model: An accurate return electrode position relative to the cochlea is implemented and modelled inside a bone ellipse roughly the size of a human head. c) Skull model: A detailed skull containing a brain volume and scalp with accurate return electrode position relative to the cochlea. The image shows the different layers of the model (sections of the scalp and skull removed to show the brain volume) with the return electrode located on the external surface of the skull. 
Table 1.

Bone resistivity values used in the simulations. The values represent human skull resistivities that were reported in literature (citations provided).

Bone resistivity

$(\Omega \mathrm{cm})$

641

2000

3125

4673

5782

6667

7943

16207

26546

\section{Reference}

1987 by Spelman and Clopton (cited in Finley et al. (1990))

Chosen for continuity

Hoekema et al. (2003)

Akhtari et al. (2002)

Tang et al. (2008)

Oostendorp et al. (2000)

Tang et al. (2008)

Akhtari et al. (2002)

Akhtari et al. (2002)
Skull sample details

Live skull (reported values) $1248-3125 \Omega \mathrm{cm}$ )

Spongy part of live skull

Dentate suture of live skull

Skull few days after excision

Standard tri-layer

Top compact bone of skull

Low compact bone of skull 


\subsection{Neuron models}

Approximately 160 neurons (depending on the basilar length of the specific cochlea) were placed in each cochlear model from the base to the apex at 5 degree intervals around the modiolus (Figure 3a). The node corresponding to the soma of each neuron was placed in the spiral ganglion region of the cochlea (Figure 1). Non-degenerate and degenerate neurons were modelled to represent the extreme cases of a healthy implanted cochlea with no neural degeneration as well as a cochlea that is assumed to have a large degree of neural degeneration. Degenerate neurons were modelled by excluding the dendritic part of the neuron and including only the somatic and axonal regions (Figure 2 and Figure 3a) (Briaire and Frijns, 2006). The potentials at these nodes were extracted from each voltage distribution profile.

To translate the potential distributions to neural excitation profiles, the resistivity matrix that describes the relationship between the injected current at the chosen electrode and the potential values at the nodes of Ranvier of the neurons was obtained. The location of the neurons along the length of the organ of Corti is represented on the $x$-axis of the resistivity map in Figure $3 b$ and the location of the nodes of Ranvier along the nerve fibres is represented on the $y$-axis. The transfer resistances were obtained by dividing the potential distribution values produced by the FE solution at the neural nodes by the injected stimulating current. These transfer resistances were then used to calculate the voltage at every node given an input current and used as inputs to a neuron model.

The responses of two physiologically based, single-fibre neuron models that are commonly employed to predict neural excitation in models of cochlear implants were compared: the HodgkinHuxley-based compartmental model of Rattay (Rattay et al., 2001c) and the generalised Schwartz- 
a)

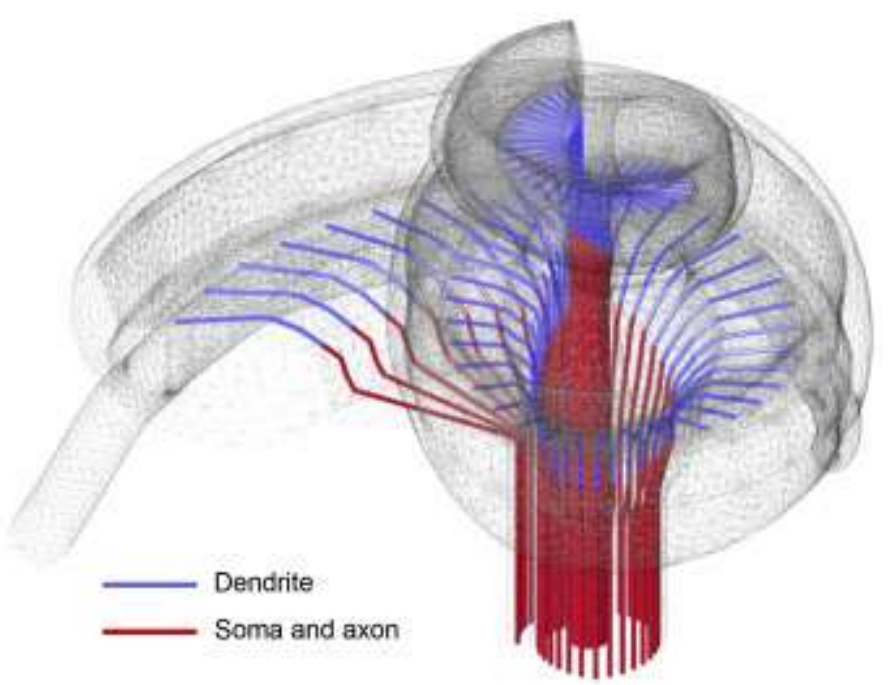

b) Transfer resistance between node and electrode [Ohm]

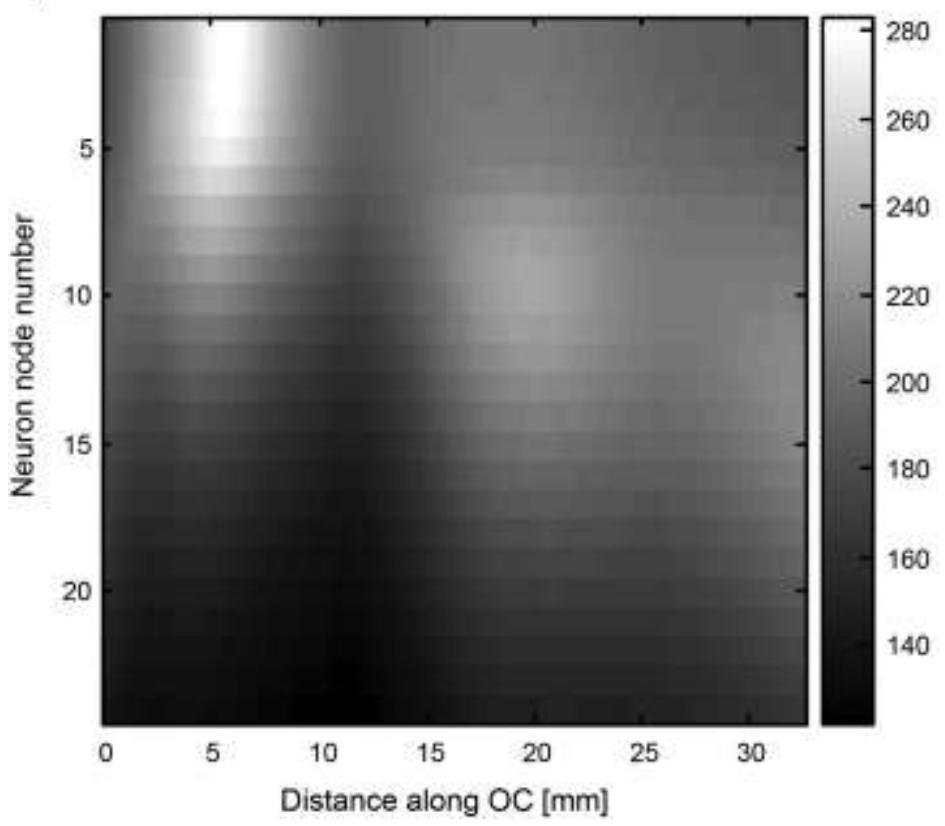

Fig. 3.

The transfer resistance between each stimulating electrode and neuron node is determined and used to calculate the input node potentials to the neural models. a) The positions of the neuron trajectories are shown here for the model of user S13R. Neurons were placed $5^{\circ}$ apart around the modiolus. Nodes were placed on these trajectories according to the nodal spacing of the neuron model used. The dendritic parts of the neurons were omitted when modelling degenerate neurons. b) The calculated transfer resistances between electrode 16 and each of the neural nodes in Ohm at a bone resistivity of $641 \Omega \mathrm{cm}$. 
Eikhof-Frijns (GSEF) model (Frijns et al., 1995). The positions of the nodes of Ranvier differ slightly according to the internodal distances associated with each model. A biphasic cathodic-first monopolar stimulus with phase duration of $77 \mu \mathrm{s}$ and an interphase gap of $0 \mu \mathrm{s}$ was used to excite the neuron model (as used in Nelson et al.). A search algorithm was employed to find the lowest current level (accurate within $1 \mu \mathrm{A}$ ) for which a neuron generates an action potential (the threshold). The thresholds of all the modelled neurons were calculated and this yielded a threshold profile.

Threshold profiles were computed for all combinations of neuron models, state of degeneration, electrode contacts, user cochlear models, head configurations and bone resistivity values. For the results reported in this study around 1000000 individual neuron thresholds were calculated.

\subsection{Bone resistivity derived from fmSTC}

Predictions of excitation spread in the present study estimates neural excitation spread directly from modelled cochlear neurons and does not consider central processing. In order to validate a model of this nature, neural excitation spread data measured at the peripheral auditory neuron level is required. This data, however, is not available and estimates from forward masking presently remains one of the only non-invasive indications of excitation spread in the live human ear.

Nelson et al. used a forward masking experiment to estimate neural excitation spread. Their forward masking paradigm was implemented on the modelled neural threshold profiles using a simplified masking model where the following assumptions were made.

1. A probe pulse is "heard" or unmasked when enough neurons are stimulated to produce a psychoacoustic threshold. The stimulus level at which this occurs is referred to as the probe threshold and was defined as the level at which a neuron population over a width of $1 \mathrm{~mm}$ along the length of the basilar membrane is stimulated (Snel-Bongers et al., 2013). For the 
probe to be heard above the masker, a section of at least $1 \mathrm{~mm}$ of neural excitation needs to extend beyond the masker's neural response.

2. Conversely, the probe pulse is masked when the non-overlapped portion of the probe response is less than $1 \mathrm{~mm}$ wide.

The same parameters as those used by Nelson et al. were used to facilitate comparison of predicted results with the measured data. The probe level was chosen as $30 \%$ of the dynamic range above threshold where the maximum comfort level was calculated as the current at which a region of 4 $\mathrm{mm}$ along the basilar membrane is stimulated (Snel-Bongers et al., 2013). A single probe electrode was used, this electrode was chosen as an electrode situated around the first half turn of every cochlea. All the electrodes were used as maskers and the level at which the probe becomes masked was determined for each masker resulting in an fmSTC from which the slope and width were measured.

The slope of each fmSTC curve was calculated by first converting the fmSTC to dB and then fitting two straight lines to the steepest parts of the fmSTC directly adjacent to the position of the minimum masking level, as was done by Nelson et al. Effectively this resulted in two exponential curves being fitted to the fmSTC curves measured in mA (Figure 4). The average slope of the two lines (in $\mathrm{dB} / \mathrm{mm}$ ) was used as the slope of the modelled fmSTC. The fmSTC width was calculated as the distance (in millimetres) between the two fitted slopes at a level of $1 \mathrm{~dB}$ above the minimum masker level (Figure 4). The widths and slopes of all the computed configurations were averaged for each bone resistivity value in each of the model configurations. The averages of the different configurations were then calculated and are summarised and compared against the Nelson et al. data (slope: $1.2 \mathrm{~dB} / \mathrm{mm}, 1 \mathrm{~dB}$ width: $4.6 \mathrm{~mm}$ ) in Figure 6 and Table 2 (discussed in the results section). 


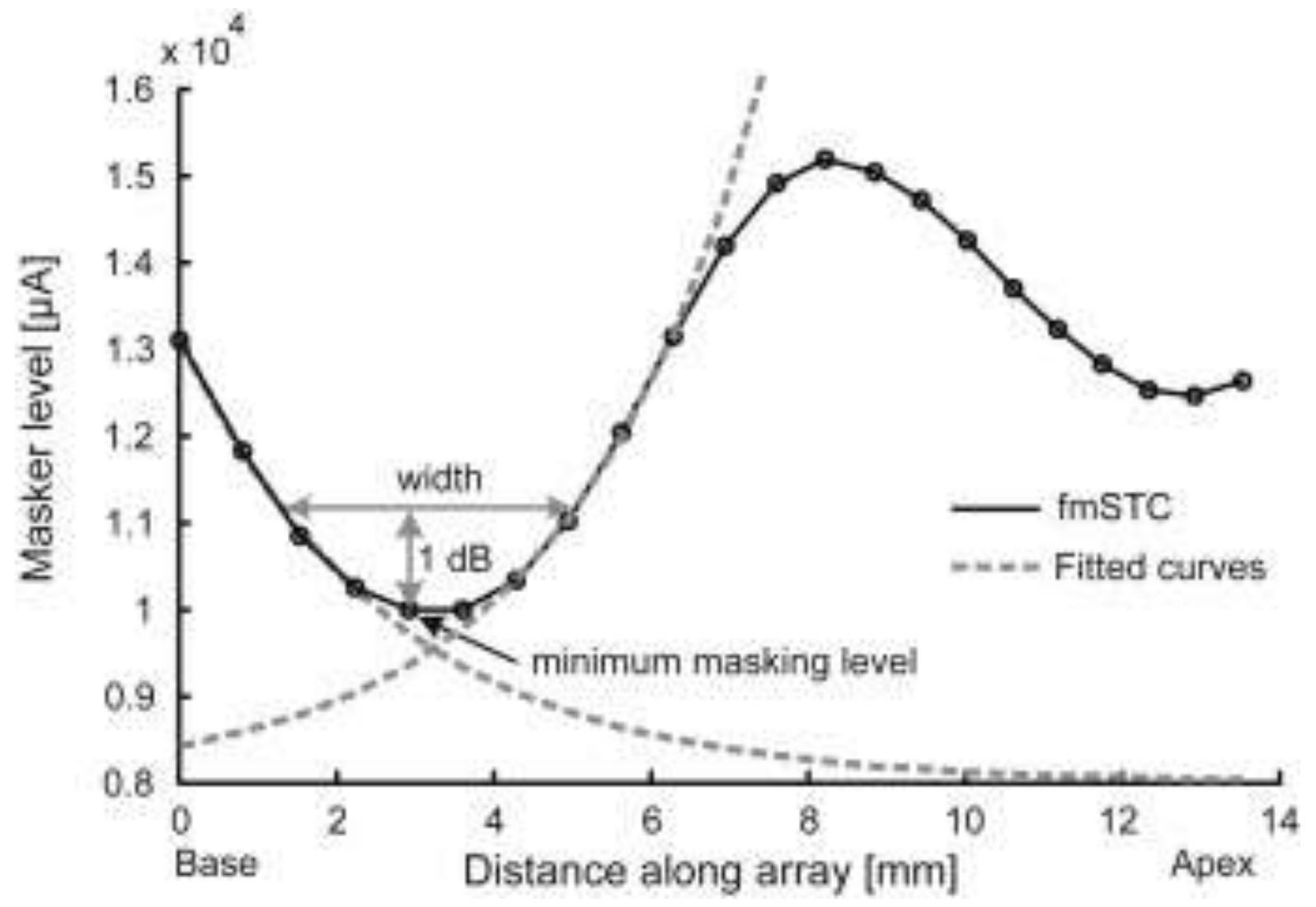

Fig. 4.

Following the methodology of Nelson et al. (2008) the slope of a fmSTC was determined by fitting two exponential curves (dashed lines) to the fmSTC adjacent to the minimum masking level. The mean of the two slopes (in $\mathrm{dB} / \mathrm{mm}$ ) was used as the slope of the profile. The $1 \mathrm{~dB}$ width was determined as the length along the electrode array between the fitted slopes at a level of $1 \mathrm{~dB}$ above the minimum masking level. 


\subsection{Modelling electric field profiles}

In Tang et al. (2011), the EFI profiles (averaged over five ears) of three stimulating electrodes (numbers 1, 9 and 15) are presented. The same electrodes were stimulated in the present models to make data comparable. Electric field profiles were generated using the five user models in each of the three head configurations using each of the specified bone resistivity values. The average electric field profile of the five user models in each configuration was used to compare to the averaged EFI profiles from literature. As the models in the present study only represent the resistive properties of the cochlear tissues and not that of the electrode tissue interface, predictions were only compared to the measurements made on the non-stimulating electrodes where there is negligible current through the electrode-tissue interface.

The electric field spread of each of these profiles was characterized in terms of its length constant $\lambda$. This was done by fitting the following exponential function (1) to each profile:

$$
V_{\text {stim_elec }}(x)=A e^{-\frac{x}{\lambda}}+C
$$

where $A$ represents the amplitude, $x$ the distance from the stimulating electrode, $\lambda$ the length constant and $C$ the DC offset of the profile of a single stimulating electrode $\left(V_{\text {stim_elec }}\right)$. The function was only fit to the first six electrodes adjacent to the stimulating electrode as cross-turn stimulation effects distort the exponential profile further along the electrode array especially at low bone resistivity values. An electrode spacing of $1.1 \mathrm{~mm}$ (Clarion HiFocus) was used when calculating the length constants. Figure 5 shows an example of the average electric field profile of a basal stimulus electrode of all the user models implemented in the skull model. The fitted exponential curve is indicated with a dashed line. 


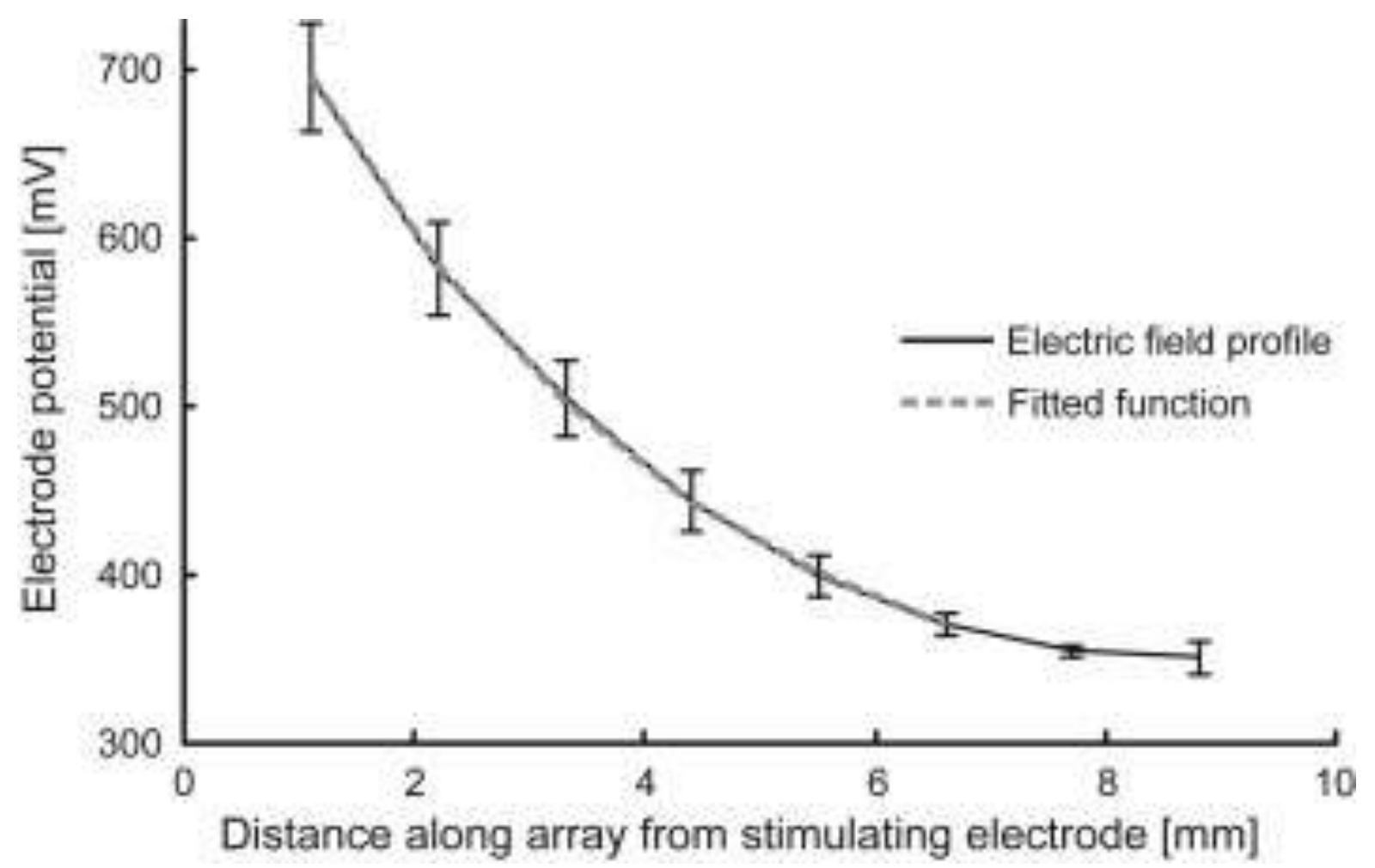

Fig. 5.

The length constant was determined by fitting the exponential function $V_{\text {stim_elec }}(x)=A \cdot \exp (-\lambda / x)+C$ to an electric field profile. Here an example is shown where the function was fit to the average electric field profile produced by stimulating the most basal electrode in all the user models. The skull head model was implemented with a bone value of $6667 \Omega \mathrm{cm}$. The function was fit to the six electrodes adjacent to the stimulating electrode to exclude cross-turn stimulation effects that occur far from the stimulating electrode. 
Only the length constant was used as the basis of comparison and not the $1 \mathrm{~dB}$ width as was used for the fmSTC data. The reason for this is that the three head configurations have different ground implementations (the infinite ground model resembles a true monopole and the other models dipoles). As all modelled potentials are calculated relative to ground, the data from each modelled head configuration will have a different $D C$ offset. This in turn makes values calculated in $d B$ incomparable. This issue is not present in the predicted neural results as the neural models use the relative potential differences between neural nodes as inputs. Different DC offsets between models do thus not affect neural model outputs.

The average length constant of the EFI profiles of the three stimulating electrodes presented in Tang et al. (2011) was calculated as $3.15 \mathrm{~mm}$. This was used as the benchmark against which the length constants of the modelled electric field profiles were compared. The predicted length constants of the three modelled head configurations are displayed as a function of bone resistivity in Figure 8 and discussed in the results section.

\section{Results}

\subsection{Bone resistivity derived from fmSTCs}

The $1 \mathrm{~dB}$ fmSTC width increases as the bone resistivity increases (Figure 6). This indicates that neural excitation becomes less focussed as bone resistivity increases. The results indicate that the $1 \mathrm{~dB}$ width varies between 0.7 and $12.6 \mathrm{~mm}$ for the infinite bone model (a), between 0.6 and $15.0 \mathrm{~mm}$ for the elliptical return electrode model (b) and between 0.6 and $15.2 \mathrm{~mm}$ for the skull model (c) as the bone resistivity increases. Nelson et al. reported an average measured $1 \mathrm{~dB}$ width value of $4.6 \mathrm{~mm}$ 
for monopolar stimulation. The bone resistivity values that produce a $1 \mathrm{~dB} \mathrm{fmSTC}$ width of $4.6 \mathrm{~mm}$ for the various model configurations are summarised in Table 2. It shows that, except for the degenerate Rattay neural models in the elliptical return electrode model, the resistivity values required by the GSEF and Rattay models are very similar for the three levels of complexity of the head model, i.e. a ratio of $91 \%$ on average between the resistivity values derived from the Rattay model to those derived for the GSEF model. The exception is for the degenerate fibres in the elliptical return electrode model where the Rattay model requires only about $31 \%$ of the resistivity value for the GSEF model to produce predictions that match the Nelson et al. data.

The average predicted fmSTC slopes also decrease as the bone resistivity increases (Figure 6). The bone resistivity values derived from the Rattay model versus the values derived from the GSEF model once again agreed to within $83 \%$ of one another for all the models but the degenerate nerve fibre models in the elliptical return electrode model. For this case the Rattay model once again only required $39 \%$ of the resistivity value required by the GSEF model to predict slopes that match the 1.2 $\mathrm{dB} / \mathrm{mm}$ slope of the measured Nelson et al. data.

If the two outlier values in the head model are ignored, the average bone resistivity value predicted over all the models is $10176 \Omega$ with a standard deviation of $1635 \Omega$. 


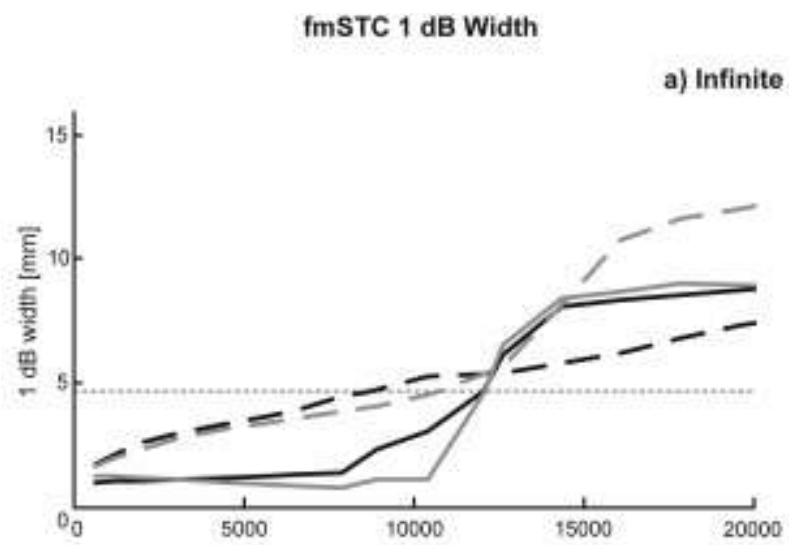

a) Infinite bone model
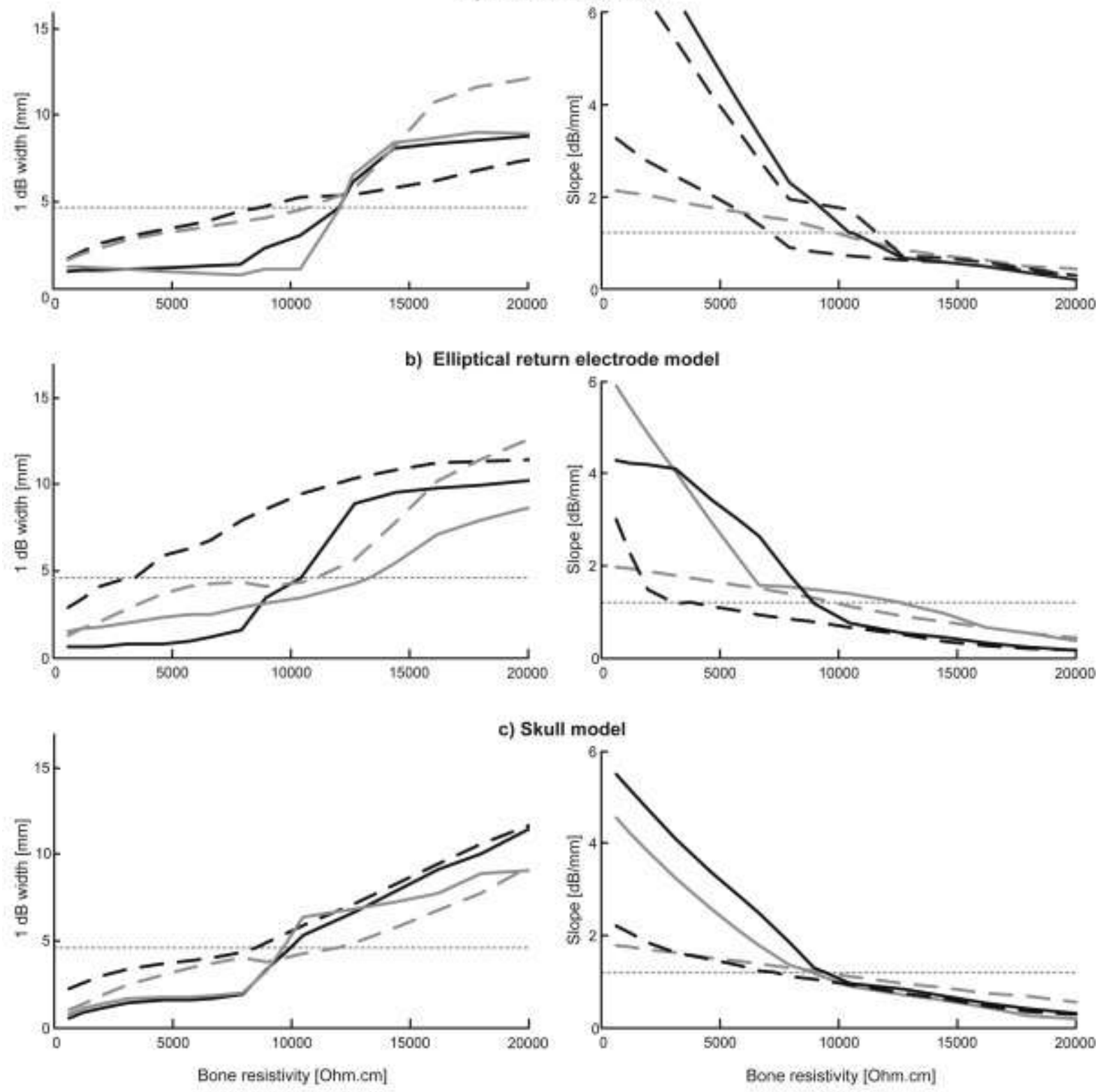

- Rattay Model Non-degenerate
- - Rattay Model Degenerate

Fig. 6.

The average predicted $1 \mathrm{~dB}$ widths $(\mathrm{mm})$ and slopes $(\mathrm{dB} / \mathrm{mm})$ of the forward masked spatial tuning curves (fmSTCs) of the three head model configurations namely the spherical model (a), elliptical model (b) and skull model (c). It is evident in all configurations that the neural excitation spread becomes wider when the bone resistivity is increased. 


\section{Table 2.}

Bone resistivity values $(\Omega \mathrm{cm})$ at which $1 \mathrm{~dB}$ widths and slopes from predicted fmSTCs from the three head models match measured data from Nelson et al. Two neuron models (Rattay and GSEF) were implemented with neurons in non-degenerated and degenerated states. The average and standard deviation of the five user cochlear models are shown in each configuration.

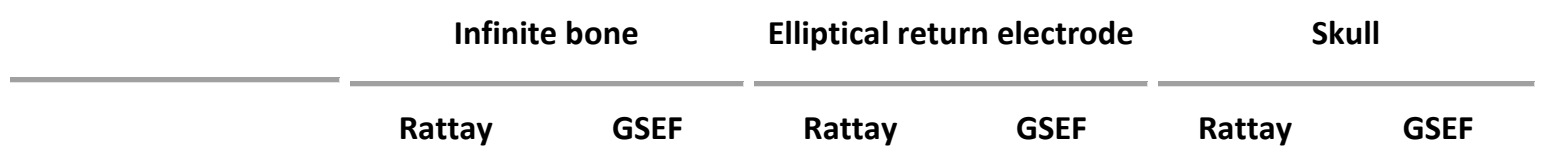
Non-degenerate Width $12016 \pm 104312222 \pm 169710475 \pm 159113342 \pm 25089988 \pm 15549649 \pm 4504$ Slope $10628 \pm 152711669 \pm 10698940 \pm 109512608 \pm 42909425 \pm 11818932 \pm 1382$

Degenerate Width $8830 \pm 2506$ Slope $7160 \pm 1325$

Average

$10426 \pm 1730$

$10808 \pm 19823450 \pm 2411 \quad 11067 \pm 18828485 \pm 203211254 \pm 2270$

$9927 \pm 2637 \quad 3772 \pm 1717$

$9174 \pm 3718$

$9287 \pm 1155$ 

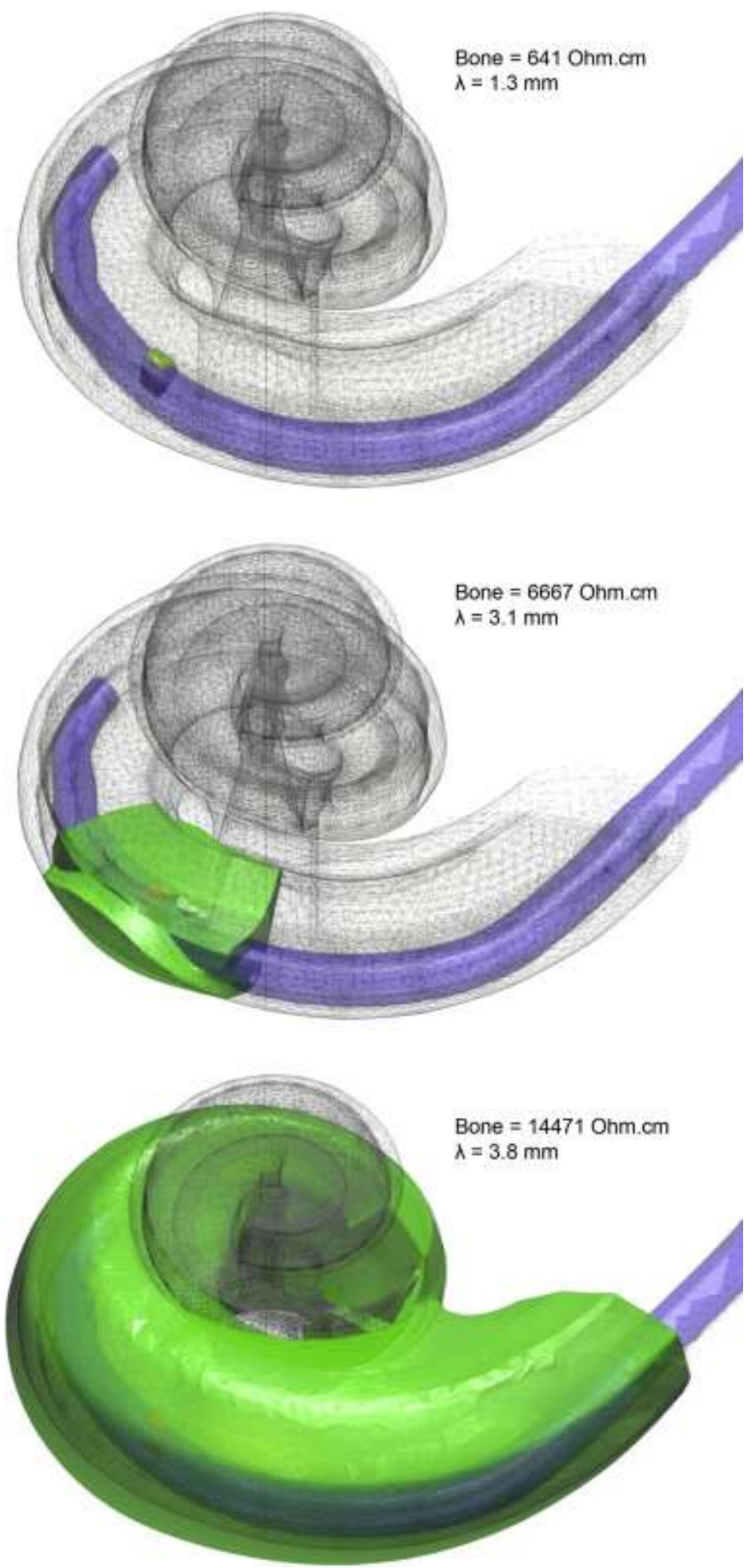

Fig. 7.

The cochlear part of an elliptical head model when stimulating a single electrode using three different bone resistivities. The green surfaces are equipotential surfaces at $0.5 \mathrm{~dB}$ below the electrode potential and indicate the widening potential field when the bone resistivity is increased. The length constant $\lambda$, approximated using the assumption of exponential decay of the potential field from the electrode $\left(y=A e^{-x / \lambda}+C\right)$, is also presented for comparison. 


\subsection{Bone resistivity derived from electrode field profiles}

Figure 7 shows the cochlear part of the elliptical return electrode head model of a single user when stimulating an electrode when three different bone resistivities are used. The green surfaces are equipotential surfaces all at $0.5 \mathrm{~dB}$ (chosen for visual clarity) below the electrode potential and visually indicate that there is a considerable change in potential field when the bone resistivity is increased.

The measured data presented by Tang et al. (2011) exhibits an average length constant of $3.15 \mathrm{~mm}$ (dashed line in Figure 8) when fitted with the exponential function (1). The bone resistivity values (interpolated from predicted data points shown in Figure 8 and summarized in table 3 ) that produce these values for the various head configurations are $6217 \Omega . c m$ for the infinite bone model, 6694

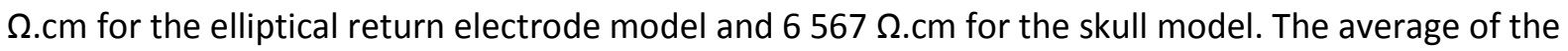
three head configurations being $6493 \Omega . \mathrm{cm}$ with a standard deviation of $2161 \Omega . \mathrm{cm}$ when including the individual values of each of the 15 models (five user models in three head configurations) in the calculations. 


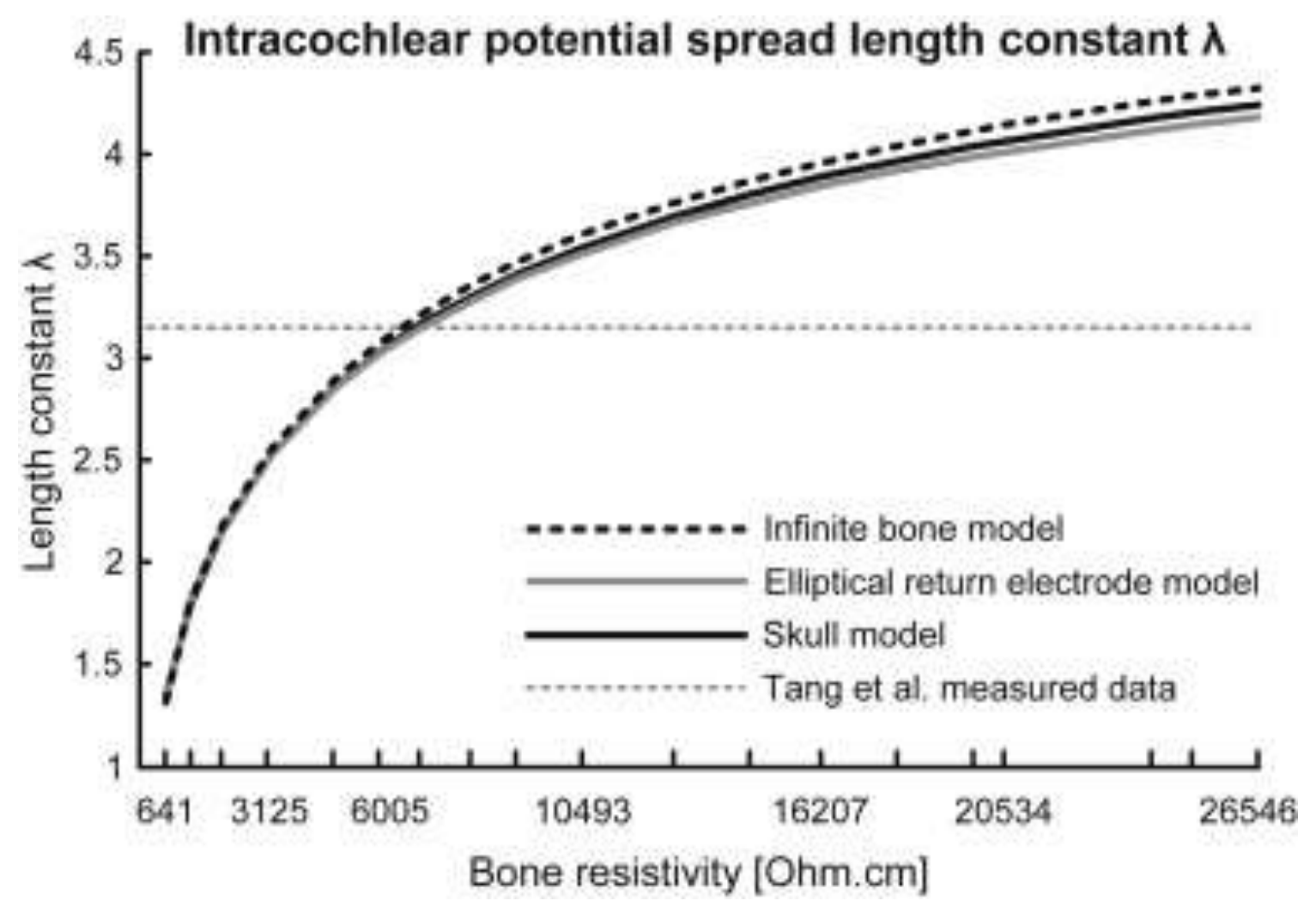

Fig. 8.

The average predicted intracochlear potential length constants, $\lambda$, of each modelled head configuration. Predicted data is compared to the length constant $(3.15 \mathrm{~mm})$ calculated from EFI measurements by Tang et al. (2011). The bone resistivity value required to match data from literature is around $6500 \mathrm{Ohm} \mathrm{cm}$ for all three head configurations indicating that potential decay inside the scala is largely unaffected by current paths to the return electrode.

\section{Table 3.}

Bone resistivity values $(\Omega \mathrm{cm})$ at which length constants $(\lambda)$ from predicted electric field profiles match the length constants of EFI measurements obtained by Tang et al. (2011). The average and standard deviation of the five user cochlear models in each head configuration are shown.

\begin{tabular}{lc}
\multicolumn{1}{c}{ Head configuration } & Bone resistivity $(\boldsymbol{\Omega} \mathrm{cm})$ \\
Infinite bone & $6217 \pm 2139$ \\
Elliptical return electrode & $6694 \pm 2462$ \\
Skull & $6567 \pm 2507$ \\
Average & $6493 \pm 2161$
\end{tabular}




\section{Discussion}

The present study set out to investigate if bone resistivity and implementation of the head structures surrounding the cochlea have a significant effect on neural excitation and intracochlear potential spread and to find a range of bone resistivity values that will allow a VC model to produce neural excitation spread predictions comparable to data measured in human implantees.

This study complements existing cochlear VC models by including various descriptions of the head volume surrounding the cochlea. It was demonstrated that the type of head model and implementation of the return electrode affects the bone resistivity value that produces predictions that match measured data. This is an important finding for modellers who wish to implement a specific level of detail into their models.

Because of the variation in human cochlear geometry (Erixon et al., 2009), cochlear models based on the geometry of five user-specific cochleae were constructed. This was done to take the effect of individual geometry variations and electrode insertions into account when predicting the average spread. The predictions from the models were averaged among the five user models to enable comparison to data from Nelson et al. and Tang et al. that were reported as the averages of multiple users. The predicted results were compared to measured fmSTC and EFI data to estimate bone resistivity values that may be used in cochlear models.

The five cochlear models were then combined with three different VC models of varying complexity of the head structure surrounding the cochlea that includes the implementation of the return electrode. In each of these 15 VC models the resistivity of the bone structures was varied and the potential spread induced by each electrode in the electrode array for each of these bone values was obtained. The voltage potentials on the electrode contacts were subsequently used to calculate electric field profiles. The voltage potentials at the locations of the neuron nodes were also applied as inputs to two physiologically based neuron models. Each of these models was used in two states 
of neural degeneration (degenerate and non-degenerate) to obtain threshold profiles for all the VC model configurations. Finally, a simple model was implemented to obtain fmSTCs from these threshold profiles.

The results of the present study indicate that the neural excitation and electric field spreads predicted by the models are significantly affected by the resistivity of the surrounding bone tissue. Wider spread (neural excitation and electric field profiles) is evident for all model configurations in both an increase in profile widths and length constants and a decrease in profile slopes as the bone resistivity increases (Figure 6 and Figure 8). The reason for this is that a higher resistivity of the bone structures shields a larger component of the stimulating current into the lower resistivity cochlear structures causing wider spread. Conversely, lower bone resistivity allows more current to leak from the cochlear structures thereby causing narrower spread of currents and thus narrower electric field and neural excitation profiles.

The bone resistivity values that predict measurements from literature differ among the three types of head models. This alludes to the fact that the potential distributions and current paths to the return electrode are dependent on the structures external to the cochlea as well as the shape and position of the return electrode. In the skull model the bone resistivity parameter represents the resistivity value of the skull, but in the infinite bone and elliptical return electrode models, the bone resistivity parameter represents a number of other structures as well. The predicted bone resistivity values for the infinite bone and elliptical return electrode models are thus single values that represent the resistivity of the compound head structure best. The variation in bone resistivity values is more pronounced in the predicted neural excitation spread than in the electric field spread. This may be an indication that the neural node potentials (which are mainly in the modiolus) are more affected by the current paths to the return electrode than the electrode potentials in the scala. 
The predicted bone resistivity values when implementing different neuron models (Rattay and GSEF) and different levels of neural degeneration have an average value of around $10000 \Omega . \mathrm{cm}$, with the exception of the degenerate Rattay model in the elliptical return electrode model. The mechanism underlying this discrepancy is speculative, but is probably related to the current path between the stimulating and return electrodes. Since the elliptical return electrode model's head structure comprises a homogeneous bone volume, the current follows a fairly direct path between the stimulating and return electrodes causing high current densities in the modiolus where the sensitive, truncated neural terminals are located. Lower neural thresholds in turn cause wider spread of excitation thereby requiring lower bone resistivity values to restrict the spread of excitation to measured values. What is evident is that truncation of the Rattay model in this head model markedly increased its sensitivity to excitation. This observation suggests that care needs to be taken when selecting a neuron model and particularly when changing the structure of a neural model, e.g. representing degeneration via truncation of the model.

Another observation is that as the bone resistivity increases, the shape of the neural excitation profile represents less of a classical threshold profile shape, which has a parabolic shape with a local minimum near the stimulating electrode, and approaches a shallower profile that has many local minima in positions not related to the stimulating electrode position nor any other obvious structures in the cochlea such as non-stimulated electrode contacts. At high resistivity values the threshold profiles from all electrodes approached a common "flat" shape. This is ascribed to the current spread in the cochlear duct being more uniform irrespective of the location of the active electrode as the insulating effects of the surrounding bone increases and forces current more longitudinally along the cochlear duct. Even though fmSTC widths and slopes could be obtained from these threshold profiles, they do not necessarily represent an accurate description of the environment in a real cochlea. Caution thus needs to be exercised when interpreting widths and slopes predicted using high bone resistivity values. It also has implications for diseases where bone density and with that possibly bone resistivity increase, e.g. in cases of osteopetrosis (Milroy and 
Michaels, 1990). The model predictions suggest that electrode discrimination and thus pitch perception may be decreased in such cases.

The bone resistivities predicted by modelling the electric field profiles are similar (average:

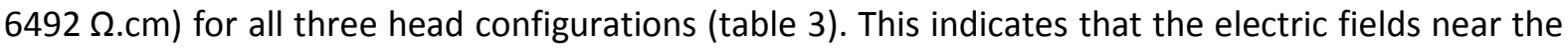
electrodes in the cochlear duct are not greatly affected by structures external to the cochlea. This is intuitively correct as the perilymph in which the electrode array is located has a low resistivity compared to other cochlear structures. The cochlear duct is thus the default path for the bulk of the current to travel through before entering other cochlear structures where it is dispersed according to these structures of higher resistivity. It was, however, found that this current path is highly dependent on the shape of the cochlear duct of the individual ear it was based on. This is clear from the high standard deviations (around $2300 \Omega . \mathrm{cm}$ ) of the predicted bone resistivity values that match measured data for the five user models within each head configuration (table 3). This highlights the importance of incorporating user-specific cochlear geometries into cochlear models when userspecific modelling results are sought.

The average bone resistivity predicted using the neural excitation spread (around $10000 \Omega . \mathrm{cm}$ ) is larger than that predicted using the intracochlear potential spread (around $6500 \Omega . \mathrm{cm}$ ). This difference in values may in part be attributed to the method used in comparing the neural excitation data to measured data. Measured data from Nelson et al. used a forward masking experiment to estimate neural excitation spread that relies on feedback given by the implanted user in a behavioural experiment. The results are thus not only a function of the neural excitation spread at the level of the cochlear neurons, but are also influenced by more central processing. As the present model does not incorporate central processes, but only peripheral single fibre responses, a difference in values may be observed. The discrepancy in the values predicted by the different neuron models (Figure 6) also suggests that the results are dependent on the type of neuron model 
used and the implementation thereof. As the neurons models are in themselves complex models relying on different assumptions and simplifications, absolute thresholds may not be predicted correctly. The bone values predicted in the present study thus compensated for these inaccuracies to produce neural excitation spreads comparable to measured data. The values recommended here are therefore only applicable to the specific neuron models used in this study and cannot necessarily be generalized to other neuron models.

In light of the variation on predicted electric field profile results, variations in neural excitation results from the different neural models and implementations as well as the fact that bone resistivity varies among individuals (Marshall et al., 1996), the following bone values are proposed for use in modelling studies. It is proposed that a bone resistivity value in the order of $10000 \Omega . c m$ be used in volume conduction models of the implanted cochlea using monopolar stimulation when neural excitation spreads are predicted (using the Rattay or GSEF neuron models) in models that incorporate any of the modelled head configurations presented here. This value does not hold where a degenerate version of the Rattay neuron model is used inside the elliptical return electrode model and a value of $3500 \Omega . \mathrm{cm}$ is proposed. When predicting electric fields in the scala of the cochlea (such as electric field profiles), a single value of $6500 \Omega . c m$ is proposed for all head configurations as head configuration has a small effect on inter scalar potentials. These values are an order higher than the bone resistivity value of $641 \Omega . \mathrm{cm}$ used in some volume conduction studies of the cochlea (Frijns et al., 1995; Finley et al., 1990; Hanekom, 2001b; Malherbe et al., 2013), whereas the values derived from the intracochlear spread is comparable to the bone resistivity values used in

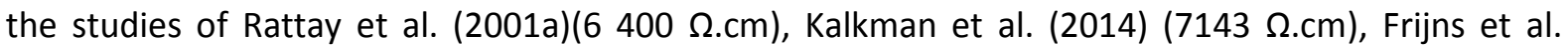

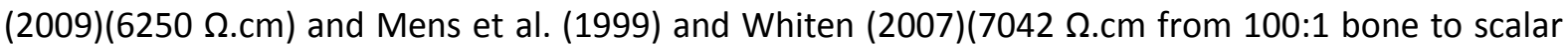
fluid conductivity ratio). 


\section{Conclusions}

(1) Neural excitation and intracochlear electric field spread are dependent on the resistivity value of the bone surrounding the cochlea with wider spread observed with an increase in bone resistivity.

(2) Neural excitation spread is highly dependent on the detail of the description of the head volume surrounding the cochlea as different bone resistivities are required in VC models to match measured spread reported in literature. The description of the head model does not considerably affect intracochlear electric fields in the highly conductive cochlear duct.

(3) A general bone resistivity of $6500 \Omega . \mathrm{cm}$ is proposed when predicting intracochlear electric field spread. When predicting neural excitation spread a default bone resistivity value of 10 $000 \Omega . c m$ is proposed for all head implementations and neural models.

However, given the sensitivity of the predicted bone resistivity values on the type of neuron model and implementation thereof as well as the geometry of a specific cochlea it is advisable to specifically optimize the bone resistivity for models that implement features that are not described in this article.

\section{Acknowledgements}

The authors wish to thank the anonymous reviewers for their many constructive comments. Financial assistance by the National Research Foundation (South Africa) is acknowledged. 


\section{References}

Akhtari, M., Bryant, H. C., Mamelak, A. N., Flynn, E. R., Heller, L., Shih, J. J., Mandelkem, M., Matlachov, A., Ranken, D. M., Best, E. D., Dimauro, M. A., Lee, R. R. and Sutherling, W. W. (2002). Conductivities of three-layer line human skull. Brain Topography, 14(3):151-167.

Bozorg Grayeli, A., Saint Yrieix, C., Imauchi, Y., Cyna-Gorse, F., Ferrary, E. and Sterkers, O. (2004). Temporal bone density measurements using CT in otosclerosis. Acta Oto-laryngologica, 124(10):1136-1140.

Briaire, J. J. and Frijns, J. H. M. (2006). The consequences of neural degeneration regarding optimal cochlear implant position in scala tympani: A model approach. Hearing Research, 214(12):17-27.

Erixon, E., Högstorp, H., Wadin, K. and Rask-Andersen, H. (2009). Variational anatomy of the human cochlea: Implications for cochlear implantation. Otology and Neurotology, 30(1):14-22.

Finley, C. C., Wilson, B. S. and White, M. W. 1990. Models of neural responsiveness to electrical stimulation. In: MILLER, J. M. and SPELMAN, F. A. (eds.) Cochlear Implants: Models of the Electrically Stimulated Ear. New York: Springer-Verlag.

Frijns, J. H. M., de Snoo, S. L. and Schoonhoven, R. (1995). Potential distributions and neural excitation patterns in a rotationally symmetric model of the electrically stimulated cochlea. Hearing Research, 87(1-2):170-186.

Frijns, J. H. M., Kalkman, R. K. and Briaire, J. J. (2009). Stimulation of the facial nerve by intracochlear electrodes in otosclerosis: A computer modeling study. Otology and Neurotology, 30(8):1168-1174.

Gabriel, S., Lau, R. W. and Gabriel, C. (1996). The dielectric properties of biological tissues: II. Measurements in the frequency range $10 \mathrm{~Hz}$ to $20 \mathrm{GHz}$. Physics in Medicine and Biology, 41(11):2251-2269.

Geddes, L. A. and Baker, L. E. (1967). The specific resistance of biological material-A compendium of data for the biomedical engineer and physiologist. Medical and biological engineering, 5(3):271-293.

Govindasamy, R. (2012). Modelling subject-specific electrically evoked auditory neural responses in the guinea pig. Masters in Engineering Dissertation, University of Pretoria.

Hanekom, T. (2001a). Modelling of the eletrode-auditory nerve fibre interface in cochlear prosthesis. $\mathrm{PhD}$ thesis, University of Pretoria.

Hanekom, T. (2001b). Three-dimensional spiraling finite element model of the electrically stimulated cochlea. Ear and Hearing, 22(4):300-315.

Haueisen, J., Ramon, C., Eiselt, M., Brauer, H. and Nowak, H. (1997). Influence of tissue resistivities on neuromagnetic fields and electric potentials studied with a finite element model of the head. IEEE Transactions on Biomedical Engineering, 44(8):727-735.

Kalkman, R. K., Briaire, J. J., Dekker, D. M. T. and Frijns, J. H. M. (2014). Place pitch versus electrode location in a realistic computational model of the implanted human cochlea. Hearing Research, 315:10-24.

Malherbe, T. K., Hanekom, T. and Hanekom, J. J. (2013). Can subject-specific single-fibre electrically evoked auditory brainstem response data be predicted from a model? Medical Engineering \& Physics, 35(7):926-936.

Marshall, D., Johnell, O. and Wedel, H. (1996). Meta-analysis of how well measures of bone mineral density predict occurrence of osteoporotic fractures. British Medical Journal, 312(7041):5.

Mens, L. H. M., Huiskamp, G., Oostendorp, T. and Van Den Broek, P. (1999). Modelling surface potentials from intracochlear electrical stimulation. Scandinavian Audiology, 28(4):249-255.

Milroy, C. M. and Michaels, L. (1990). Pathology of the otic capsule. Journal of Laryngology and Otology, 104(2):83-90.

Nelson, D. A., Donaldson, G. S. and Kreft, H. (2008). Forward-masked spatial tuning curves in cochlear implant users. Journal of the Acoustical Society of America, 123(3):1522-1543. 
Rattay, F., Leao, R. N. and Felix, H. (2001a). A model of the electrically excited human cochlear neuron. II. Influence of the three-dimensional cochlear structure on neural excitability. Hearing Research, 153(1-2):64-79.

Rattay, F., Lutter, P. and Felix, H. (2001c). A model of the electrically excited human cochlear neuron I. Contribution of neural substructures to the generation and propagation of spikes. Hearing Research, 143:43-63.

Rush, S. and Driscoll, D. A. (1968). Current distribution in the brain from surface electrodes. Anesthesia and Analgesia, 47(6):717-723.

Snel-Bongers, J., Briaire, J. J., Van Der Veen, E. H., Kalkman, R. K. and Frijns, J. H. M. (2013). Threshold levels of dual electrode stimulation in cochlear implants. JARO - Journal of the Association for Research in Otolaryngology, 14(5):781-790.

Tang, Q., Benítez, R. and Zeng, F. G. (2011). Spatial channel interactions in cochlear implants. Journal of Neural Engineering, 8(4).

Whiten, D. M. (2007). Electro-anatomical Models of the Cochlear Implant. PhD, Massachusetts Institute of Technology. 